\title{
. \\ SUMOylation Regulates BmNPV Replication by Moderating PKIP Intracellular Localization
}

\author{
Rui Shen ${ }^{1, \dagger}$, Dingding Lü ${ }^{2,+}$, Guanyu Chen ${ }^{1}$, Mengjin Liu ${ }^{1}$, Shiqi Pu ${ }^{1}$, Yiling Zhang ${ }^{1,3}$, Qiang Wang ${ }^{1,3}$, \\ Ping Qian ${ }^{1,3}$ and Xudong Tang ${ }^{1,3, * \mathbb{D}}$
}

1 School of Biotechnology, Jiangsu University of Science and Technology, Zhenjiang 212018, China; shenrui20202020@163.com (R.S.); szysri@163.com (G.C.); liumengjin666@163.com (M.L.); psqshiqi@163.com (S.P.); zhangyiling008@126.com (Y.Z.); wqiang523@just.edu.cn (Q.W.); qianping@just.edu.cn (P.Q.)

2 School of Nursing, Zhenjiang College, Zhenjiang 212028, China; ludingding@zjc.edu.cn

3 Sericulture Research Institute, Chinese Academy of Agricultural Science, Zhenjiang 212018, China

* Correspondence: xudongt@just.edu.cn

+ These authors contributed equally to this work.

check for updates

Citation: Shen, R.; Lü, D.; Chen, G.; Liu, M.; Pu, S.; Zhang, Y.; Wang, Q.; Qian, P.; Tang, X. SUMOylation Regulates BmNPV Replication by Moderating PKIP Intracellular Localization. Processes 2022, 10, 261. https://doi.org/10.3390/pr10020261 Academic Editor: Keping Chen

Received: 21 December 2021

Accepted: 23 January 2022

Published: 28 January 2022

Publisher's Note: MDPI stays neutral with regard to jurisdictional claims in published maps and institutional affiliations.

Copyright: (C) 2022 by the authors. Licensee MDPI, Basel, Switzerland. This article is an open access article distributed under the terms and conditions of the Creative Commons Attribution (CC BY) license (https:// creativecommons.org/licenses/by/ $4.0 /)$.

\begin{abstract}
SUMOylation is a reversible covalent process between a small ubiquitin-like modifier (SUMO) and its target protein and has become a crucial regulator of protein functions. Here, we report that Bombyx mori nucleopolyhedrovirus (BmNPV) may take advantage of the host SUMOylation system to enhance its own replication, similar to many other viruses. Both the knockdown of BmSUMO by RNAi and chemical blocking by ginkgolic acid both impaired BmNPV replication. Using site mutation and pull-down assays, we found that lysine K70 of the protein kinase-interacting protein (PKIP), which is conserved in all Alphabaculoviruses, was modified by SUMO. Mutation of K70 in PKIP led to its translocation from the cytoplasm to the nucleus. Knockout and rescue experiments showed that the rescue of PKIP mutant virus with wild-type PKIP restored BmNPV replication to the normal level, but this was not true for the K70R mutation. Altogether, these results show that SUMOylation of PKIP plays a key role in BmNPV replication.
\end{abstract}

Keywords: SUMO; silkworm; Baculovirus; BmNPV; PKIP

\section{Introduction}

Post-translational protein modifications (PTMs), such as ubiquitinoylation, phosphorylation, neddylation, and glycosylation, enable cells to control the function of proteins. A small ubiquitin-like modifier (SUMO) was characterized during a study on the effect of covalent modifications on the nuclear import of RanGAP1 [1,2]. SUMO interacts with the substrate proteins in two ways. One way is by covalently attaching SUMO to the $\varepsilon$-amino group of lysine residues of the substrate protein to regulate several biological processes, such as transcription regulation, cell cycle process, innate immunity modulation, and DNA damage repair [3-5]. Covalently binding to the substrate protein involves at least four enzymes, including an E1-activating enzyme complex (SAE1/SAE2), a unique E2-conjugating enzyme (Ubc9), and some E3 ligases (such as PIAS), which are conjugated to its substrates through the lysine $(\mathrm{K})$ at the consensus motif $(\psi \mathrm{KxE})$, where $\psi$ is a hydrophobic amino acid [6-8]. The non-covalent interaction of SUMO and its substrate occurs through SUMO-interacting motifs, which affect its activity by non-covalent attachment. The short motif $(\mathrm{V} / \mathrm{I} / \mathrm{L}) \mathrm{X}(\mathrm{V} / \mathrm{I} / \mathrm{L})(\mathrm{V} / \mathrm{I} / \mathrm{L})$ is considered the necessary motif in substrate proteins for SUMO interaction $[9,10]$.

SUMOylation is used by hosts or viruses to inhibit or benefit viral replication. During infection, viruses, such as filoviruses, picornaviruses, herpesviruses, adenoviruses, papillomaviruses, and orthomyxoviruses, can interact with the host's SUMOylation system to improve viral replication [11]. Some viral proteins are known to have the ability to regulate 
the host cellular environment by manipulating the SUMOylation system, resulting in a more favorable environment for virus propagation or preventing the host's antiviral immune system from activating $[12,13]$. Baculoviruses are large DNA viruses that infect insects and could be used to produce recombinant proteins or control pest insects [14]. Bombyx mori nucleopolyhedrovirus (BmNPV) is a member of the Alphabaculovirus genus of the Baculoviridae family, and it infects the economically important insect silkworm Bombyx mori. Studies have shown that BmNPV and its host have complex interactions [15-17].Although many proteins encoded by baculoviruses have been reported to have post-translational protein modifications, including phosphorylation, ubiquitination, glycosylation, and acetylation, only a few observations were made regarding SUMO and baculoviruses. Liu et al. found that the fusion of engineered SUMO to target proteins can increase its expression in the baculovirus/insect cell system and Langeresis et al. showed that the baculovirus/insect cell system can be used to produce SUMOylated proteins [18,19].

A baculovirus relies on host cells to facilitate its replication. We found that many silkworm proteins are modified by BmSUMO [20]. We report that the SUMOylation system of host cells is critical for baculovirus replication. These findings provide a new target for the treatment of diseases caused by BmNPV in B. mori.

\section{Materials and Methods}

\subsection{Silkworm and Cell Line}

Silkworm strain p50 (Dazao) was raised at the Sericultural Research Institute, Chinese Academy of Agricultural Sciences (Zhenjiang, China). The larvae were fed mulberry leaves (Morus sp.) and kept at $25 \pm 1{ }^{\circ} \mathrm{C}$ with $70-85 \%$ relative humidity and a $12 \mathrm{~h}$ light/dark photoperiod. On the third day, the 5th instar larvae were dissected, and different tissues were collected and stored at $-80^{\circ} \mathrm{C}$ for RNA extraction. The BmN (Bombyx mori $\mathrm{N}$ ) cell line was maintained in TC-100 supplemented with 10\% fetal bovine serum (Invitrogen, Carlsbad, USA).

\subsection{Amplification of BmSUMO by Reverse Transcription Quantitative Polymerase Chain Reaction $(R T-q P C R)$}

Total RNAs from BmN cells were extracted using TRIzol reagent (Invitrogen, Carlsbad, USA) according to the manual. To obtain the first strand of cDNA, $1 \mu \mathrm{g}$ of total RNA was reverse-transcribed into cDNA with Reverse Transcriptase M-MLV (Takara, Dalian, China) with oligo dT primers. The BmSUMO gene was amplified using the primer BmSUMO.

Tissues of B. mori, including the silk gland, Malpighian tubule, midgut, head, blood, fat body, sericterium, epidermis, testis, and ovary, were dissected and subjected to total RNA extraction using TRIzol reagent. RT-qPCR was performed with SYBR Green PCR Master Mix (Takara, Dalian, China) and the primer qBmSUMO under the following conditions: $95^{\circ} \mathrm{C}$ for $2 \mathrm{~min}$, followed by 45 cycles of $95^{\circ} \mathrm{C}$ for $15 \mathrm{~s}$ and $60{ }^{\circ} \mathrm{C}$ for $40 \mathrm{~s}$. Each reaction was repeated three times. The results were normalized to the expression level of $B$. mori glyceraldehyde-3-phosphate dehydrogenase (GAPDH) using the primer qGAPDH.

To detect SUMOylation genes in BmN cells after BmNPV infection, BmN cells $\left(1 \times 10^{6}\right)$ were seeded in 6-well plates and infected with BmNPV (T3 strain) at a multiplicity of infection (MOI) of 10. At $0,1.5,3,6,12,24,48$, and $72 \mathrm{~h}$ post-infection (hpi), the cells were collected, and RT-qPCR was performed using the primers listed in Table S1.

For analysis of gene transcription by RT-qPCR, three independent experiments with three technical replicates were performed. A relative quantitative method $\left(2^{-\Delta \Delta C t}\right)$ and Student's $t$-test were used to evaluate differences in the expression.

\subsection{Phylogenetic Analysis}

The amino acid sequences of SUMO from different species were downloaded from GenBank. The GenBank accession numbers are as follows: Bombyx mori (NP_001037410), Antheraea yamamai (BAD66842), Drosophila melanogaster (NP_477411), Aedes aegypti (EAT32712), Anopheles gambiae (XP_321390), Tribolium castaneum (XP_970781), Apis mellifera (XP_623227), 
Culex quinquefasciatus (XP_001861568), Acyrthosiphon pisum (ABD91520), Mus musculus SUMO-2 (NP_579932), Homo sapiens SUMO-1 (BC008450), Homo sapiens SUMO-2 (BC006462), Homo sapiens SUMO-3 (XM_009805), Homo sapiens SUMO-4 (NP_001002255), Arabidopsis thaliana (X99609), Saccharomyces cerevisiae (NP_010798), Nasonia vitripennis (XP_001599647), Ixodes scapularis (EEC07197), Xenopus laevis (NP_001079759), Bos Taurus (NP_001069917), Equus caballus (XP_001499576), Gallus gallus (NP_001072966), Ornithorhynchus anatinus (XP_001517656), and Danio rerio (NP_001003422). Sequence alignment was performed using ClustalW on the website www.expasy.org (accessed on 12 October 2021), and homology shading was performed using GeneDoc software.

\subsection{Knockdown of BmSUMO and BmUBC9 in BmN cells}

To knockdown BmSUMO and BmUBC9, siRNA was designed using the online software BLOCK iT $^{\mathrm{TM}}$ RNAi Designer based on the open reading frame of BmSUMO. The siRNA sequences for BmSUMO knockdown were $5^{\prime}$-GCUUAUUGUGAUAGAGCAG-3' and $5^{\prime}-$ CUGCUCUAUCACAAUAAGC- $3^{\prime}$. The siRNA sequences for BmUBC9 knockdown were $5^{\prime}$-AAGCCUACACAAUUUAUUGUC- ${ }^{\prime}$ and $5^{\prime}$-GACAAUAAAUUGUGUAGGCUU- ${ }^{\prime}$. For the negative control, which was a scrambled siRNA, the sense and antisense sequences were 5'-GCUUGUUAUAGGAGAUCAG-3' and 5'- CUGAUCUCCUAUAACAAGC-3', respectively. For transfection, $4 \mu \mathrm{g}$ of each siRNA oligo was sequentially mixed with $200 \mu \mathrm{L}$ serum-free TC-100 media and $4 \mu \mathrm{L}$ of NeofectTM DNA transfection reagent (NEOFECT, Beijing, China). After $30 \mathrm{~min}$, the mixture was added to the well $\left(1 \times 10^{6}\right.$ cells/well). The cells were then cultured at $28^{\circ} \mathrm{C}$ for $24 \mathrm{~h}$. The RNAi efficiency of BmSUMO and $U b c 9$ was assayed by RT-qPCR.

\subsection{Virus Infection and Ginkgolic Acid Treatment}

To monitor the replication of BmNPV, a recombinant BmNPV ( $\left.\mathrm{V}^{\mathrm{Bm}-\mathrm{EGFP}}\right)$ with eGFP was constructed [21]. For RNAi, BmN cells were infected $24 \mathrm{~h}$ after transfection with $\mathrm{V}^{\mathrm{Bm}-\mathrm{EGFP}}$ at an MOI of 10 . After $1 \mathrm{~h}$ of infection, the cells were washed three times with serum-free medium, and fresh medium was added. The time point was defined as $0 \mathrm{hpi}$. At $48 \mathrm{hpi}$, the cells were collected for viral DNA copy analysis [22]. The supernatants were also collected for the determination of budded virus (BV) titer using TCID $_{50}$. For ginkgolic acid treatment, BmN cells $\left(1 \times 10^{6}\right.$ cells) were seeded in 6-well plates and infected with $\mathrm{V}^{\mathrm{Bm}-\mathrm{EGFP}}$ at an MOI of 10 in the presence of ginkgolic acid (Sigma Aldrich, St. Louis, USA) at a final concentration of 1,5 , and $10 \mu \mathrm{M}$. DMSO was used as a negative control. At certain time points after infection, cells and supernatants were collected for DNA copy and BV titer analyses, as described above.

\subsection{Plasmid Generation}

GFP and BmSUMO were cloned into PIZV5-His to generate a PIZ-GFP-SUMO-fused expression vector. The HA-tagged PKIP was amplified using the primer set HA-PKIP. Next, HA-PKIP was cloned into PIZV5-His at the EcoRI and Xhol sites to generate a PIZ-HA-PKIP vector. K67 and K70 mutations were introduced in the PIZ-HA-PKIP construct using the primers PKIPK67R and PKIPK70R, respectively, according to the instructions (QuikChange Site-Directed Mutagenesis Kit; Agilent Technologies, Beijing, China). The sequences of the resulting PIZ-HA-PKIPK67R and PIZ-HA-PKIPK70R were confirmed by sequencing.

GFP and PKIP were sequentially cloned into PIZV5-His to generate a PIZ-GFP-PKIP vector. The same primers, PKIPK67R and PKIPK70R, were used to create PIZ-GFPPKIPK67R and PIZ-GFP-PKIPK70R mutations, respectively.

\subsection{Pull-Down and Western Blotting Assays}

For the pull-down assay, $2 \times 10^{7}$ cells were co-transfected with PIZ-HA-PKIP and PIZGFP-SUMO, PIZ-HA-PKIP and PIZ-HA-PKIPK67R, PIZ-HA-PKIP and PIZ-HA-PKIPK70R, or PIZ-GFP-SUMO and PIZ empty vector (negative control). At $72 \mathrm{~h}$ after transfection, the cells were collected and washed with ice-cold PBS ( $\mathrm{pH}$ 7.4). An-ti-GFP pull-down assays 
were performed with a $\mu$ MACS GFP Isolation Kit (Miltenyi Biotec, Bergisch Gladbach, Germany) according to the user manual.

The resulting proteins were separated by SDS-PAGE and transferred onto a polyvinylidene difluoride membrane. eGFP-epitope (Beyotime, Beijing, China) and anti-rabbit HRPconjugated secondary antibodies (Pierce, Rockford, MI, USA) were used to detect the reactive band(s). The results were visualized using an ECL detection system.

\subsection{Construction of Recombinant Viruses}

Escherichia coli BW25113, which harbors BmBacmid, was used to generate the PKIP-KO recombinant viruses as previously described [23]. Briefly, a fragment containing the cat gene cassette $(\mathrm{CmR})$ and the PKIP flanking regions were amplified by PCR from plasmid pRADZ3 using the PKIP-KO primer set (Table S1). Next, the linear fragment was electrotransformed into E. coli BW25113, resulting in the replacement of the $342 \mathrm{bp}$ segment of PKIP with $C m R$ to generate a PKIP-KO recombinant bacmid, bPKIP-KO.

To generate a PKIP rescue plasmid, wild-type PKIP and PKIP (K70R) were inserted into the cloning sites of the transfer vector $\mathrm{pFBI}-\mathrm{PH}-\mathrm{GFP}$. The resulting plasmids were named pFBI-PKIP Re1 and pFBI-PKIP Re2, respectively. Then, a helper plasmid encoding the transposase pMON7124 and bPKIP-KO was transformed into DH10B cells. The resulting cells were transformed with the transfer plasmids pFBI-PH-GFP, pFBI-PKIP Re1, and pFBI-PKIP Re2 to generate a BmBacmid with PKIP disrupted ( $\mathrm{V}^{\mathrm{PKIP}-\mathrm{KO}}$ ) and two rescued BmBacmid ( $\mathrm{V}^{\mathrm{PKIP} R e 1}$ and $\left.\mathrm{V}^{\mathrm{PKIP}} \mathrm{Re} 2\right)$, respectively. A control BmBacmid $\left(\mathrm{V}^{\mathrm{wt}}\right)$ was constructed by transforming pFBI-PH-GFP into DH10B cells harboring the wild-type BmBacmid. All BmBacmids contained a gfp reporter gene under the control of the ie-1 promoter. BmBacmid DNA was purified with QIAGEN large-construct kit (QIAGEN, Hiden, Germany) and quantified by spectrometry.

\subsection{Virus Growth Curve Analysis}

For viral growth curves, $\mathrm{BmN}$ cells were transfected in triplicates with $1.0 \mu \mathrm{g}$ bacmid DNA with lipofectin (Thermo Fisher Scientific, former Savant, Waltham, MA, USA). At the indicated time points, the supernatants of each sample containing BV were harvested. The titers of all supernatants of $\mathrm{BmN}$ cells were determined using the TCID ${ }_{50}$ method [24].

\section{Results}

\subsection{Characterization of the BmSUMO Gene}

The cDNA sequence and amino acid sequence of BmSUMO were aligned against the silkworm genomic sequence using NCBI BLAST (www.ncbi.nlm.nih.gov, accessed on 12 October 2021) to study the genomic organization of the silkworm SUMO gene. There was only one copy of the BmSUMO gene in the whole B. mori genome, located on chromosome 23. The coding region of BmSUMO is interrupted by two introns. When the amino acids of BmSUMO were aligned with the SUMO proteins of yeast and humans, the conserved Cterminal diglycine cleavage/attachment site was observed (Figure S1). BmSUMO contains 91 amino acids with the highest identity, $96.7 \%$, with A. yamamai SUMO and $39.6 \%$ identity with yeast Smtp3. There are four SUMO proteins in the human genome: SUMO 1-4. SUMO-2 and SUMO-3 share high sequence identity (about 87\%) with each other, while sharing low identity with SUMO-1 and SUMO-4 (about 44\%). It was reported that the nematode SUMO is more similar to SUMO-1, whereas Drosophila SUMO is more similar to SUMO-2/3 [25-27]. BmSUMO is also more similar to vertebrate SUMO-2/3, sharing $67 \%$ identity; in contrast, it has only $51.6 \%$ identity with human SUMO-1. RT-qPCRs were performed to analyze the transcription patterns of BmSUMO in different tissues of the fifth instar larvae. BmSUMO is differently expressed in different tissues, with the lowest expression level in the Malpighian tubule and highest in the ovary and testis (Figure S2). This result is similar to that of a previous observation [28]. 


\subsection{Expression of SUMOylation-Related Genes in B. mori after BmNPV Challenge}

To monitor SUMOylation genes after BmNPV challenge, BmN cells were infected with BmNPV, and the expressions of BmSUMO, BmUBC9 (XM_028187026.1), BmSAE1 (NM_001047020.1), BmSAE2 (NM_001145333.1), and BmPIAS (XM_038017812.1) were analyzed by RT-qPCR. As shown in Figure 1, the expressions of all genes gradually but modestly increased after BmNPV challenge. The attachment of SUMO to target proteins can modulate the functionality of hundreds of proteins. Notably, the innate immune system is closely regulated by the SUMOylation system [29-31]. As an important part of the immune system, the components of the NF- $\mathrm{KB}$ and interferon pathways are SUMOylated to modulate the anti-pathogen system, such as Pellino 1, TANK, IK $\beta \alpha$, and NEMO of the NF-kB pathway, as well as IRF8, IRF7, PMLIV, and IRF3 of the interferon pathway [30]. It was reported that the expression of BmSUMO increases after an immune challenge. The expression of BmRelA and CecropinB1 decreased significantly after the knockdown of $B m S U M O$ in vivo, indicating that BmSUMO may regulate the innate immune response [7]. Thus, the induced expression of genes of the SUMOylation system indicated that there are some connections between BmNPV infection, SUMOylation, and innate immunity.

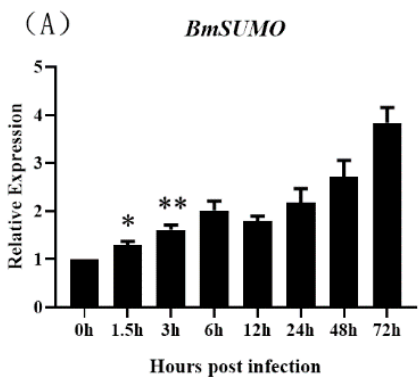

(C)

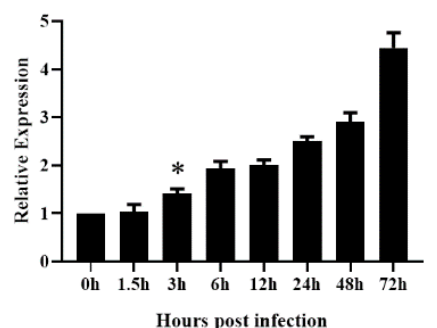

(B)

BmUBC9

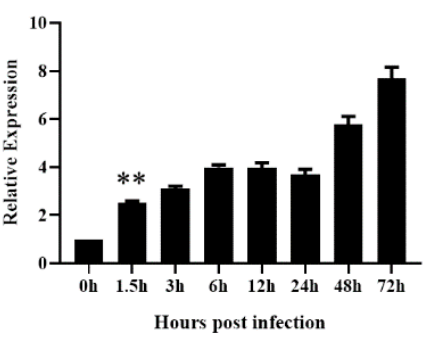

(D)

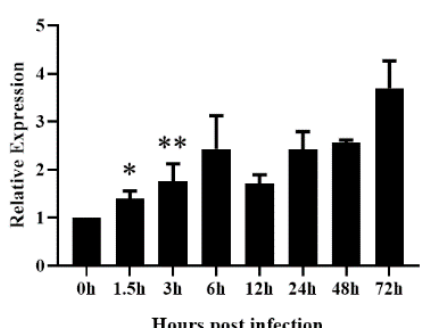

Hours post infection

Figure 1. Expression of SUMOylation-related genes BmSUMO (A), BmUBC9 (B), BmSAE1 (C), and $B m S A E 2$ (D) after BmNPV infection. BmN cells were infected with BmNPV (T3 strain) at a MOI of 10. At $0,1.5,3,6,12,24,48$, and $72 \mathrm{hpi}$, the cells were collected and subjected to reverse-transcription quantitative polymerase chain reaction analysis. Statistical analysis was applied with SPSS software. One-way ANOVA with Tukey's post-test was used. Significant differences are indicated by asterisks $\left({ }^{*} p<0.05 ;{ }^{* *} p<0.01\right)$.

\subsection{Knockdown of BmSUMO and BmUBC9 Inhibited BmNPV Replication}

To further identify the relationship between BmSUMO and BmNPV replication, endogenous expression of BmSUMO was knocked down by siRNA. The results showed that endogenous BmSUMO expression was knocked down by approximately 30\% (Figure 2A) which led to a significant decrease in viral DNA copies and BV titer (Figure 2B,C). The knockdown of the BmUBC9 led to similar results (Figure 2D-F). 
(A)

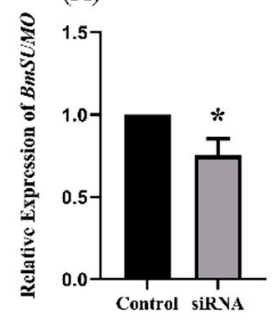

(D)

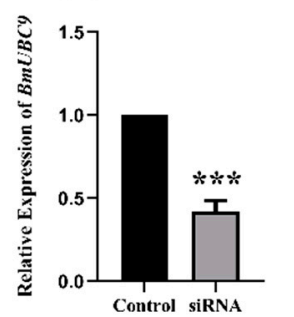

(B)

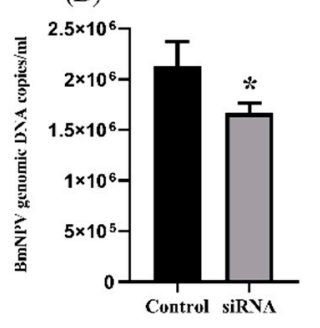

(E)

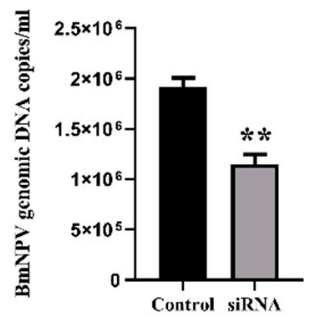

(C)

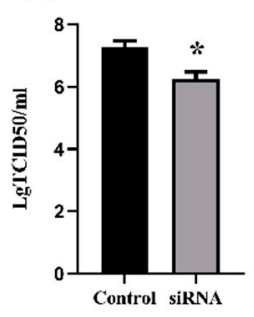

(F)

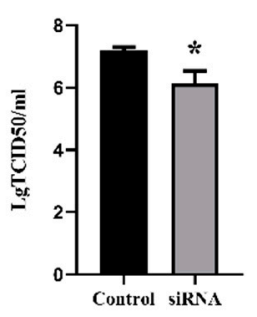

Figure 2. Knockdown of BmSUMO and BmUBC9 inhibited BmNPV replication. (A) Expression level analysis of BmSUMO at $24 \mathrm{~h}$ after transfection with siRNA. (B,C) DNA copies and budded virus titer of BmNPV in the control and BmSUMO-knockdown cells at 48 hpi. (D-F) The same as BmSUMO but with $B m U B C 9$. The error bars indicate the SDs of averages from three independent experiments with three technical replicates. Statistical analysis was applied with SPSS software. One-way ANOVA with Tukey's post-test was used. Significant differences are indicated by asterisks $\left({ }^{*} p<0.05 ;{ }^{* *} p<0.01\right.$; *** $p<0.001)$.

\subsection{Ginkgolic Acid Suppressed BmNPV Replication}

To confirm the relationship between SUMOylation and BmNPV replication, we used ginkgolic acid, which can directly bind SAE1 and inhibit the formation of the SAE1-SUMO complex to inhibit SUMOylation without affecting in vivo ubiquitination in BmN cells [32]. BmN cells were treated with 1,5, and $10 \mu \mathrm{M}$ of ginkgolic acid and then infected with $\mathrm{V}^{\mathrm{Bm} \text {-EGFP }}$. At $48 \mathrm{hpi}$, the cells were observed under a fluorescence microscope. As shown in Figure $3 \mathrm{~A}$, the fluorescence intensity of the ginkgolic-acid-treated group reduced in a concentration-dependent manner and was significantly lower than that of the negative control group. The BV titer and genomic DNA copies also decreased under ginkgolic acid treatment (Figure 3B,C).

\subsection{PKIP of BmNPV Was SUMOylated at K70}

We found that some proteins from BmNPV may be modified by SUMO, such as PKIP, which is an essential protein related to BV production [33,34]. A SUMO modification site prediction using SUMOplot software showed that PKIP contains two possible SUMO modification sites: K67 and K70. K70 is highly conserved compared to other nuclear polyhedrosis viruses (Figure 4A). To confirm the interaction between SUMO and PKIP, BmN cells were co-transfected with GFP-SUMO and wild-type HA-PKIP or mutant HAPKIP (K67R and K70R). GFP pull-down assays showed the binding between GFP-SUMO and HA-PKIP. The K70R mutation of PKIP disabled its SUMO-binding ability, whereas K67R retained its activity. The results indicated that PKIP is SUMOylated at the K70 site. 
(A)
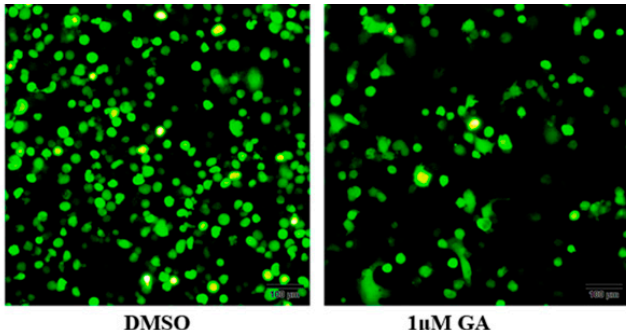

1нM GA

(B)

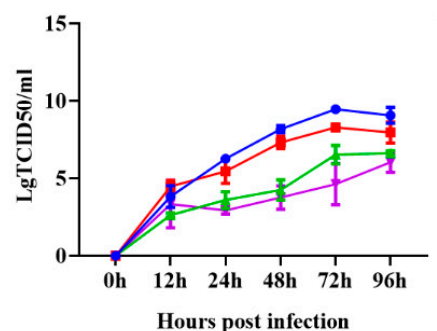

$\rightarrow$ DMSO

\# GA $5 \mu \mathrm{M}$

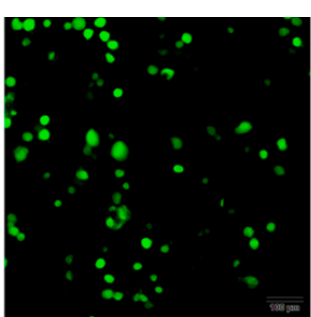

5нM GA

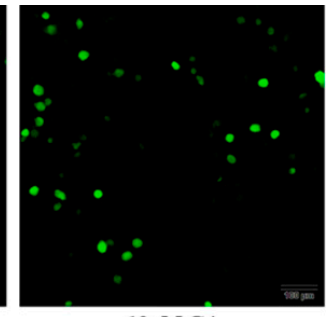

$10 \mu \mathrm{M} \mathrm{GA}$

(C)

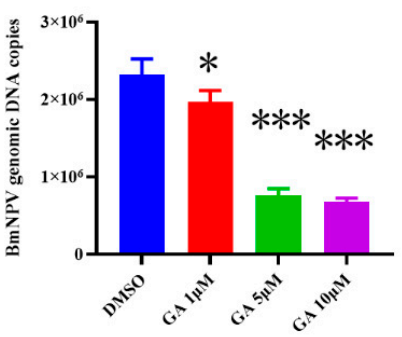

Figure 3. Ginkgolic acid (GA) inhibited BmNPV replication. (A) Fluorescence of $V^{B m-E G F P}$-infected $\mathrm{BmN}$ cells under treatment with different concentrations of ginkgolic acid at $48 \mathrm{hpi}$. Bar $=100 \mu \mathrm{m}$. (B) The budded virus titer curve of $\mathrm{V}^{\mathrm{Bm} \text {-EGFP }}$ under ginkgolic acid treatment. (C) DNA copies of BmNPV at 48 hpi. The error bars indicate the SDs of averages from three independent experiments with three technical replicates. Statistical analysis was applied with SPSS software. One-way ANOVA with Tukey's post-test was used. Significant differences are indicated by asterisks $\left({ }^{*} p<0.05 ; * * *<0.001\right)$.

(A)

$\mathrm{BmNPV}$

PxMNPV

AcNPV

RoMNP

TaNPV

ToNPV

MvNPV

CpNPY

CONPV

DpNPV

CdNPV

DkNPV

OpMNPV

CrNPV

CfMNP

CrMNPV

ApNPV

ApNPV

HcNPV

JNPV

(B)

PKIP+SUMO

PKIP

$$
\mathrm{K} 67 \mathrm{~K} 70
$$

IAADIKGQVEQLELVNQYLDAPKSEKLEFVYNCSDLDINEEDLKSLCLTKNIAYFTERYN IAAD IKGQVEQLELVVQYLGAPESEKLDFVYDCSDLDINEKDLKSLCLTKNIAYFTQKYN IAADIKGQVEQLELVNQYLGAPESEKLDFVYDCSDLDINEKDLKSLCLTKNIAYFTQKYN IAADIKGQVEQLELVNQYLGAPESEKLDFVYDCFDLD INEKDLKSLCLTKNIAYFTQKYN IAAD IKGQVEQLELVNQYLGAPESEKLDFVYDCSDLD INEKDLKSLCLTKNIAYFTQKYN VAADIKGEIEQLELVYQYMNLPDSEKLDFVYDCEDLDIDKKDLKSLCLTKNIAYFSQKYN IAADIRGQVEQLELVSQYLDAPESEKLNFVNDCSDLDINEEDL----LTKNITYFTQKYN LAADIHGTMELLEAALAIADASDNEKLNFIYDCFDLDFEIEDLRALCNNKNFAYFSEKYN MADIHGOIFOIEALLSVAHAADEEKLEFAKDCSDLDIDPAELVYCKOLN SYFATKYO AAD IYGQVEQLEALLSVARAPHADKLEPABCSDLDISODLOLCARSDTFATKYO LAADIYOU LAAEIHGQVELEALLSVARASDADKREFARDCSDLDIDAQDLQRMCASNEPTYFAAKYD LAADIYGQVEQLEALLSVARAPHADKLEFARDCSDLDINLQDLQLMCARSDLTYFATKYE VAADLYGSIEHIEVLQMFIDATAAIKLKFAHDFTDLDIDKTDVKMLHDTKKIDYFSIKYN LAAEIHGQVEQLEALLSVARASDADKREFARDCSDLDIDAQDLQRMCASNEPTYFAAKYD LAADIHGQVEQLEALLSVARASDADKLEFARDCSDLDIDSQDLQRMCARNDLAYFATKYE LAADIHGQIEQMEALLSVAHKADEEKLEFAKDCSDLDIDPAELSRVCKQFDLSYFANKYE LAADIHGQIEQVEALLSIAHADDEEKLEFAKDCSDLDFDSAELICVCEQSDLSYFAVKYD LADIYGQIFQLEALLSVAHAPDSEKLEFARDCSDLDMDPAVLARVCAGD-AAHFATKYG AADINGQIEOLEALISVADASDADKL KFVRDLSDIDIDTOEL NPVCARNDLTYFYSKYE LAADIYGQVEQLEALLSVARASNADKFEFVRDWSDLDIDVQDLRRICARNDSTYFATKYD 


\subsection{Localization of PKIP Was Related to SUMOylation}

The PKIP of AcMNPV was detected throughout the cells and predominantly localized to the inner nuclear membrane of the infected cells at $18 \mathrm{hpi}$, suggesting that the cellular localization PKIP might be dependent on its SUMOylation. SUMOylation is known to affect the cellular localization of numerous SUMO targets, including several viral proteins $[12,35,36]$. Therefore, we speculated that the cellular localization of PKIP might be affected by SUMOylation. To this end, wild-type PKIP or mutant PKIP (K67R and K70R) was fused and transiently expressed with eGFP. As shown in Figure 5, the wild-type and mutant PKIP (K67R) localized in the whole cells, whereas PKIPK70R predominantly localized in the cytoplasm. The results indicated that the translocation of PKIP from the cytoplasm to the nucleus is dependent on its SUMOylation state.

DAPI

GFP-PKIP
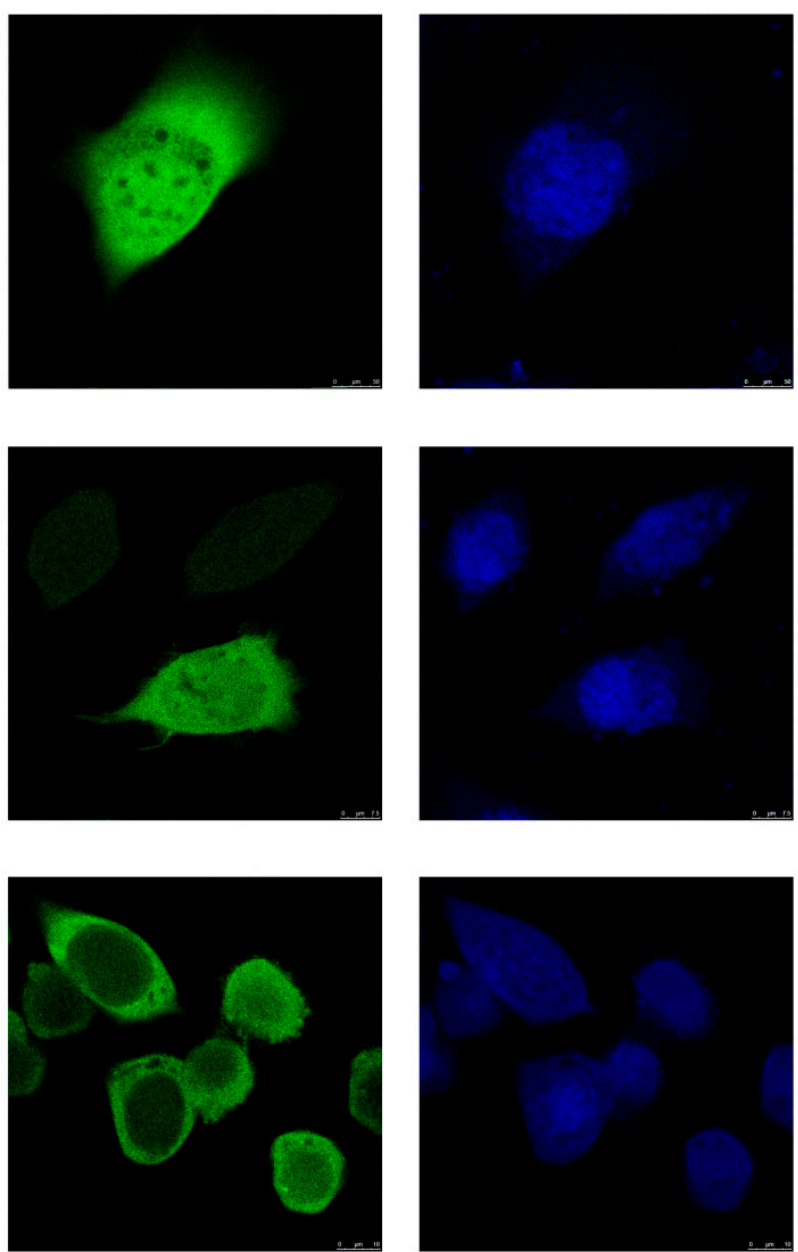

Merge
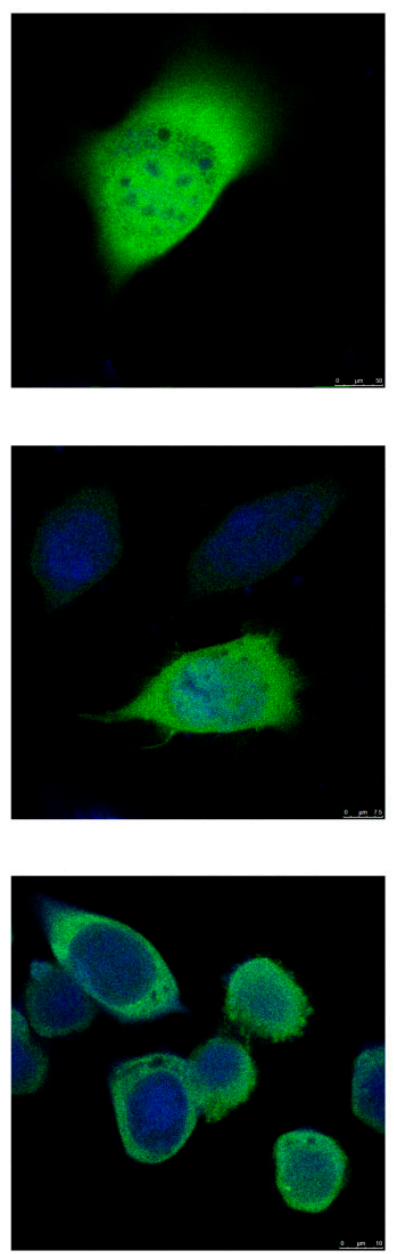

Figure 5. Subcellular location of PKIP was related to SUMOylation. BmN cells were transfected with wild-type or mutant PKIP (K67R and K70R). At $48 \mathrm{~h}$ after transfection, the cells were observed under a confocal laser fluorescence microscope. The nuclei were stained with 4',6-diamidino-2phenylindole (DAPI).

\subsection{SUMOylation of PKIP Was Essential for BmNPV Replication}

To directly address the connection between PKIP SUMOylation and BmNPV replication, a series of PKIP-knockout and repair viruses was constructed. When PKIP was knocked out, the replication of BmNPV significantly decreased. Repair with wtPIKP and vPKIPRe1 restored viral replication up to a level similar to that of the wild-type, whereas that with the K70R mutant PKIP-vPKIPRe2 did not (Figure 6). These results indicated that the SUMOylation of PKIP is essential for BmNPV replication. 

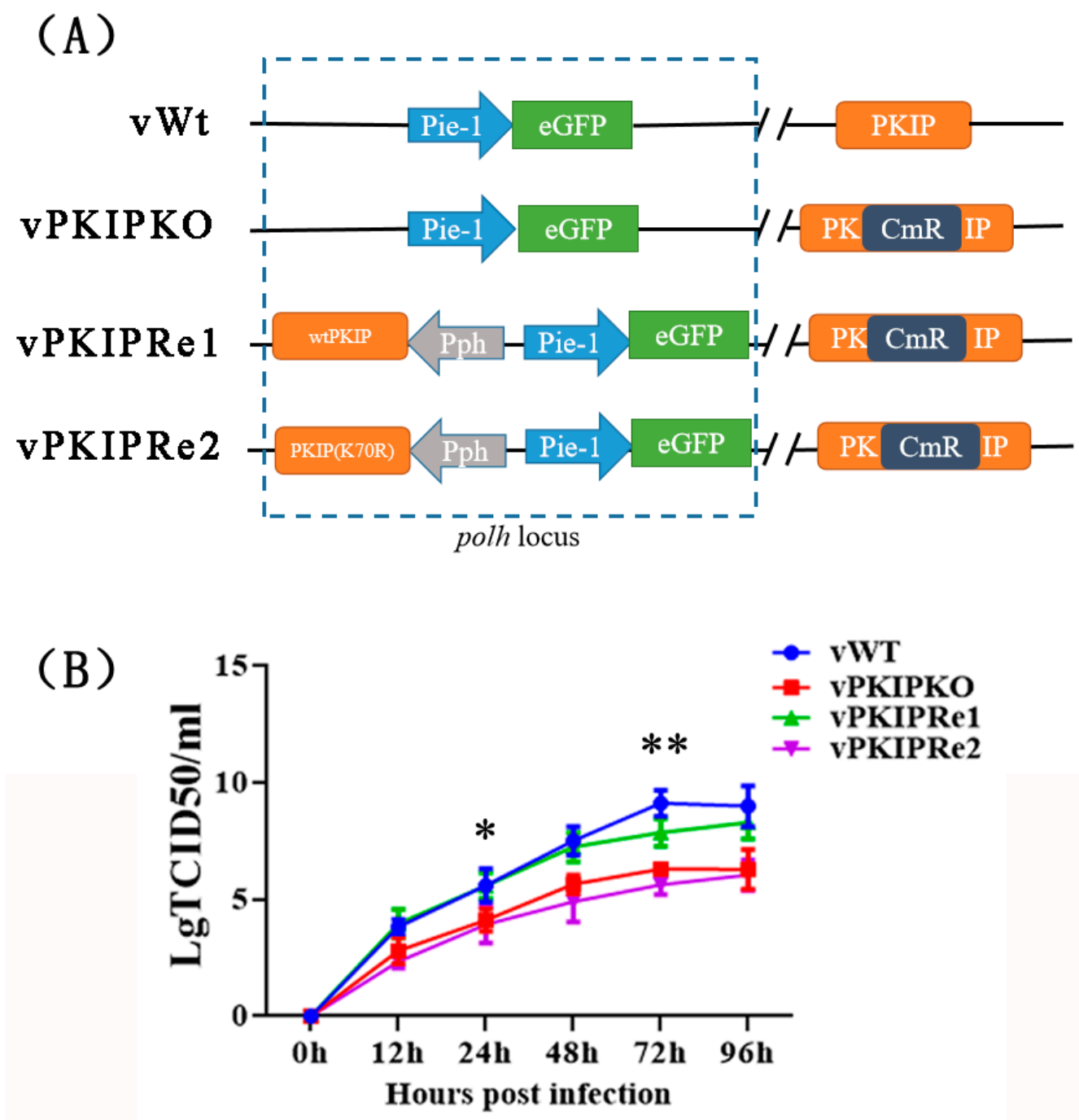

Figure 6. SUMOylation of PKIP is important to BmNPV replication. (A) Wild-type and mutant PKIP (K70R) were transformed to the polh locus of the PKIP-KO virus to generate different repair viruses. (B) The BV titer curves of each recombinant virus were determined. $\mathrm{V}^{\mathrm{Wt}}$, virus without PKIP knockout; vPKIP, virus with PKIP knockout; vPKIPRe1, PKIP-knockout virus rescued with wild-type PKIP; vPKIPRe2, PKIP-knockout virus rescued with K70R-mutated PKIP. The error bars indicate the SDs of averages from three independent experiments with three technical replicates. Statistical analysis was applied with SPSS software. One-way ANOVA with Tukey's post-test was used. Significant differences are indicated by asterisks $\left({ }^{*} p<0.05 ; * *<0.01\right)$.

\section{Discussion}

SUMOylation is an important reversible and highly dynamic post-translational modification involved in many cellular processes, including the control of gene transcription, cell signaling, and embryonic development. We showed here that the SUMO modification system of $B$. mori is important for the replication of BmNPV, similar to many other viruses. When RNA interference or compounds (ginkgolic acid) were applied to reduce or block the SUMO system of the host cells, the replication of BmNPV was significantly inhibited.

Different viruses use different mechanisms to take advantage of the SUMO modification system [37]. Some viral proteins, such as IE1 of CMV; BEFL1, LF2, and EBNA3C of EBV; K-bZIP and LANA1 of KSHV; and E3 of vaccinia virus, are modified by SUMO to activate their function $[7,28]$. Alternatively, some viral proteins, such as Gam1, ICP0, and K-Rta, affect the global SUMOylation status of the infected cells. Moreover, the same virus can use different proteins to interact with the host SUMOylation system, such as the p6, IN, UL44, and pp71 proteins of HIV [38,39]. During the identification of SUMOylated proteins 
in B. mori, we also found that some proteins from BmNPV can be modified by SUMO, such as FP25K, PKIP, P40, and IE-0. As PKIP is associated with the nucleocapsids of BV and contributes to the optimal production of $\mathrm{BV}$, which is consistent with the results of SUMO RNAi and GA inhibition, PKIP was chosen for further confirmation. We found that PKIP is modified at K70, which is conserved in the entire group I baculovirus, but not at K67 as predicted by SUMOplot software.

It was reported that PKIP of AcMNPV is distributed in both the cytoplasm and nuclei of virus-infected cells and that its dynamic localization is important for viral replication. We observed here that the transport of PKIP from the cytoplasm to the nucleus is lost when K70 is mutated. Eventually, the K70 mutation of PKIP reduces viral replication. The PKIP may stimulate the activity of protein kinase 1 (PK1), which is the structural protein of both BV and ODV, and is essential for the nucleocapsid assembly and viral very late gene expression $[40,41]$. Since the nucleocapsid assembly and viral very late gene expression all happen within the nucleus, the PKIP has to be transported from the cytoplasm to the nucleus. The transport of proteins from the cytoplasm into the nucleus is complicated and some proteins depend on nuclear localization signals (NLS) while others are not dependent on NLS. Additionally, nuclear transport is precisely regulated by PTMs such as phosphorylation/dephosphorylation, arginine methylation, acetylation, and so on [42]. SUMOylation can also modulate the subcellular localization of substrate proteins. For example, the influenza A virus nucleoprotein is essential for the intracellular trafficking of nucleoproteins and for viral growth $[43,44]$. The SUMOylation of Zika virus and Dengue virus NS5 proteins directs their nuclear localization [45]. The DENV NS5 protein conserved SUMO-interacting motifs (SIM) sites are required for both NS5 SUMOylation and nuclear localization, while the ZIKV SIM site was only necessary for nuclear localization. For PKIP, the NLS sequence and the relationship between NLS and K70 remain to be classified. The possible rule of SUMOylated PKIP being transported from the cytoplasm to the nucleus may be due to the recognition of SUMOylated PKIP by the nuclear transportation system of cells.

\section{Conclusions}

In summary, these results identified SUMOylation as a positive regulator of BmNPV replication in silkworms. The regulatory effect is based on the K70 modification of PKIP. These findings can contribute to the design of new antivirals capable of modulating BmNPV replication.

Supplementary Materials: The following supporting information can be downloaded at: https: / / www.mdpi.com/article/10.3390/pr10020261/s1, Figure S1. The alignment of SUMO proteins between species. Figure S2. The expression pattern of BmSUMO in different tissues of silkworm, Bombyx mori. Figure S3. Knockdown of UBC9 inhibited viral DNA replication of Bombyx mori nucleopolyhedrovirus (BmNPV). Table S1. Primers used in PCR and RT-qPCR.

Author Contributions: Investigation, R.S. and S.P.; Methodology, D.L. and Y.Z.; Software, G.C.; validation, M.L.; Writing, X.T.; Formal analysis, Q.W.; Resources, P.Q. All authors have read and agreed to the published version of the manuscript.

Funding: This work was supported by the Natural Science Foundation of Jiangsu Province (BK20190244), the Science and Technology Innovation Fund Project of Zhenjiang City (NY2021020, SH2021066), and Jiangsu Province Universities "Blue Project".

Institutional Review Board Statement: Not applicable.

Informed Consent Statement: Not applicable.

Data Availability Statement: The data used to support the findings of this study are available from the corresponding author upon request.

Acknowledgments: The authors would like to thank the Zhongyuan Shen for the support and critically reviewing the manuscript. 
Conflicts of Interest: All authors declare no conflicts of interest.

\section{References}

1. Saitoh, H.; Pu, R.; Cavenagh, M.; Dasso, M. RanBP2 associates with Ubc9p and a modified form of RanGAP1. Proc. Natl. Acad. Sci. USA 1997, 94, 3736-3741. [CrossRef] [PubMed]

2. Mahajan, R.; Delphin, C.; Guan, T.; Gerace, L.; Melchior, F. A small ubiquitin-related polypeptide involved in targeting RanGAP1 to nuclear pore complex protein RanBP2. Cell 1997, 88, 97-107. [CrossRef]

3. Baz-Martinez, M.; El Motiam, A.; Ruibal, P.; Condezo, G.N.; de la Cruz-Herrera, C.F.; Lang, V.; Collado, M.; San Martin, C.; Rodriguez, M.S.; Munoz-Fontela, C.; et al. Regulation of Ebola virus VP40 matrix protein by SUMO. Sci. Rep. 2016, 6, 37258. [CrossRef] [PubMed]

4. $\quad$ Dohmen, R.J. SUMO protein modification. Biochim. Biophys. Acta 2004, 1695, 113-131. [CrossRef]

5. Su, C.I.; Tseng, C.H.; Yu, C.Y.; Lai, M.M.C. SUMO Modification Stabilizes Dengue Virus Nonstructural Protein 5 To Support Virus Replication. J. Virol. 2016, 90, 4308-4319. [CrossRef]

6. Hay, R.T. Decoding the SUMO signal. Biochem. Soc. Trans. 2013, 41, 463-473. [CrossRef]

7. Muller, S.; Dejean, A. Viral immediate-early proteins abrogate the modification by SUMO-1 of PML and Sp100 proteins, correlating with nuclear body disruption. J. Virol. 1999, 73, 5137-5143. [CrossRef]

8. Schwienhorst, I.; Johnson, E.S.; Dohmen, R.J. SUMO conjugation and deconjugation. Mol. Gen. Genet. 2000, 263, 771-786. [CrossRef]

9. Song, J.; Durrin, L.K.; Wilkinson, T.A.; Krontiris, T.G.; Chen, Y. Identification of a SUMO-binding motif that recognizes SUMOmodified proteins. Proc. Natl. Acad. Sci. USA 2004, 101, 14373-14378. [CrossRef]

10. Song, J.; Zhang, Z.; Hu, W.; Chen, Y. Small ubiquitin-like modifier (SUMO) recognition of a SUMO binding motif: A reversal of the bound orientation. J. Biol. Chem. 2005, 280, 40122-40129. [CrossRef]

11. Mattoscio, D.; Segre, C.V.; Chiocca, S. Viral manipulation of cellular protein conjugation pathways: The SUMO lesson. World. J. Virol. 2013, 2, 79-90. [CrossRef] [PubMed]

12. El Motiam, A.; Vidal, S.; Seoane, R.; Bouzaher, Y.H.; Gonzalez-Santamaria, J.; Rivas, C. SUMO and Cytoplasmic RNA Viruses: From Enemies to Best Friends. Adv. Exp. Med. Biol. 2020, 1233, 263-277. [CrossRef] [PubMed]

13. Mattoscio, D.; Medda, A.; Chiocca, S. Recent Highlights: Onco Viral Exploitation of the SUMO System. Curr. Issues Mol. Biol. 2020, 35, 1-16. [CrossRef] [PubMed]

14. Wang, M.; Hu, Z. Cross-talking between baculoviruses and host insects towards a successful infection. Philos. Trans. R. Soc. Lond B. Biol. Sci. 2019, 374, 20180324. [CrossRef]

15. Huang, H.; Wu, P.; Zhang, S.; Shang, Q.; Yin, H.; Hou, Q.; Zhong, J.; Guo, X. DNA methylomes and transcriptomes analysis reveal implication of host DNA methylation machinery in BmNPV proliferation in Bombyx mori. BMC. Genomics 2019, $20,736$. [CrossRef]

16. Mao, F.; Lei, J.; Enoch, O.; Wei, M.; Zhao, C.; Quan, Y.; Yu, W. Quantitative proteomics of Bombyx mori after BmNPV challenge. J. Proteomics 2018, 181, 142-151. [CrossRef]

17. Xue, J.; Qiao, N.; Zhang, W.; Cheng, R.L.; Zhang, X.Q.; Bao, Y.Y.; Xu, Y.P.; Gu, L.Z.; Han, J.D.; Zhang, C.X. Dynamic interactions between Bombyx mori nucleopolyhedrovirus and its host cells revealed by transcriptome analysis. J. Virol. 2012, 86, 7345-7359. [CrossRef]

18. Langereis, M.A.; Rosas-Acosta, G.; Mulder, K.; Wilson, V.G. Production of sumoylated proteins using a baculovirus expression system. J. Virol. Methods. 2007, 139, 189-194. [CrossRef]

19. Liu, L.; Spurrier, J.; Butt, T.R.; Strickler, J.E. Enhanced protein expression in the baculovirus/insect cell system using engineered SUMO fusions. Protein. Expr. Purif. 2008, 62, 21-28. [CrossRef]

20. Tang, X.; Fu, X.; Hao, B.; Zhu, F.; Xiao, S.; Xu, L.; Shen, Z. Identification of sumoylated proteins in the silkworm Bombyx mori. Int. J. Mol. Sci. 2014, 15, 22011-22027. [CrossRef]

21. Wang, X.Y.; Shao, Z.M.; Chen, Q.Y.; Xu, J.P.; Sun, X.; Xu, Z.P.; Li, M.W.; Wu, Y.C. Knockdown of BmTCP-1beta Delays BmNPV Infection in vitro. Front. Microbiol. 2019, 10, 578. [CrossRef] [PubMed]

22. Toufeeq, S.; Wang, J.; Zhang, S.Z.; Li, B.; Hu, P.; Zhu, L.B.; You, L.L.; Xu, J.P. Bmserpin2 Is Involved in BmNPV Infection by Suppressing Melanization in Bombyx mori. Insects 2019, 10, 399. [CrossRef] [PubMed]

23. Tang, X.D.; Xu, Y.P.; Yu, L.L.; Lang, G.J.; Tian, C.H.; Zhao, J.F.; Zhang, C.X. Characterization of a Bombyx mori nucleopolyhedrovirus with Bmvp80 disruption. Virus Res. 2008, 138, 81-88. [CrossRef] [PubMed]

24. Xu, W.; Wang, H.; Liu, H.; Wu, X. Bombyx mori nucleopolyhedrovirus F-like protein Bm14 is a cofactor for GP64-Mediated efficient infection via forming a complex on the envelope of budded virus. Virology 2020, 539, 61-68. [CrossRef]

25. Jones, D.; Crowe, E.; Stevens, T.A.; Candido, E.P. Functional and phylogenetic analysis of the ubiquitylation system in Caenorhabditis elegans: Ubiquitin-conjugating enzymes, ubiquitin-activating enzymes, and ubiquitin-like proteins. Genome Biol. 2002, 3, research0002.1. [CrossRef]

26. Su, H.L.; Li, S.S. Molecular features of human ubiquitin-like SUMO genes and their encoded proteins. Gene 2002, 296, 65-73. [CrossRef]

27. Abed, M.; Bitman-Lotan, E.; Orian, A. The Biology of SUMO-Targeted Ubiquitin Ligases in Drosophila Development, Immunity, and Cancer. J. Dev. Biol. 2018, 6, 2. [CrossRef] 
28. Xu, H.P.; Hao, W.; He, D.; Xu, Y.S. Smt3 is required for the immune response of silkworm, Bombyx mori. Biochimie 2010, 92, 1306-1314. [CrossRef]

29. Adorisio, S.; Fierabracci, A.; Muscari, I.; Liberati, A.M.; Ayroldi, E.; Migliorati, G.; Thuy, T.T.; Riccardi, C.; Delfino, D.V. SUMO proteins: Guardians of immune system. J. Autoimmun. 2017, 84, 21-28. [CrossRef]

30. Liu, J.; Qian, C.; Cao, X. Post-Translational Modification Control of Innate Immunity. Immunity 2016, 45, 15-30. [CrossRef]

31. Schmidt, N.; Domingues, P.; Golebiowski, F.; Patzina, C.; Tatham, M.H.; Hay, R.T.; Hale, B.G. An influenza virus-triggered SUMO switch orchestrates co-opted endogenous retroviruses to stimulate host antiviral immunity. Proc. Natl. Acad. Sci. USA 2019, 116, 17399-17408. [CrossRef] [PubMed]

32. Fukuda, I.; Ito, A.; Hirai, G.; Nishimura, S.; Kawasaki, H.; Saitoh, H.; Kimura, K.; Sodeoka, M.; Yoshida, M. Ginkgolic acid inhibits protein SUMOylation by blocking formation of the E1-SUMO intermediate. Chem. Biol. 2009, 16, 133-140. [CrossRef] [PubMed]

33. Lai, Q.; Xu, L.; Wang, Y.; Luo, W.; Zhu, L.; Yuan, M.; Wu, W.; Yang, K. AcMNPV PKIP is required for hyperexpression of very late genes and involved in the hyperphosphorylation of the viral basic protein P6.9. Virus Res. 2020, 279, 197889. [CrossRef] [PubMed]

34. Lai, Q.; Zhu, L.; Xu, L.; Yuan, M.; Wu, W.; Yang, K. AcMNPV PKIP is associated with nucleocapsid of budded virions and involved in nucleocapsid assembly. Virus Res. 2019, 268, 27-37. [CrossRef]

35. Jan Fada, B.; Kaadi, E.; Samrat, S.K.; Zheng, Y.; Gu, H. Effect of SUMO-SIM Interaction on the ICP0-Mediated Degradation of PML Isoform II and Its Associated Proteins in Herpes Simplex Virus 1 Infection. J. Virol. 2020, 94, e00470-20. [CrossRef] [PubMed]

36. Palacios, S.; Perez, L.H.; Welsch, S.; Schleich, S.; Chmielarska, K.; Melchior, F.; Locker, J.K. Quantitative SUMO-1 modification of a vaccinia virus protein is required for its specific localization and prevents its self-association. Mol. Biol. Cell 2005, 16, $2822-2835$. [CrossRef]

37. Lowrey, A.J.; Cramblet, W.; Bentz, G.L. Viral manipulation of the cellular sumoylation machinery. Cell Commun. Signal. 2017, 15, 27. [CrossRef]

38. Campbell, M.; Izumiya, Y. Post-Translational Modifications of Kaposi's Sarcoma-Associated Herpesvirus Regulatory ProteinsSUMO and KSHV. Front. Microbiol. 2012, 3, 31. [CrossRef]

39. Kim, E.T.; Kim, Y.E.; Kim, Y.J.; Lee, M.K.; Hayward, G.S.; Ahn, J.H. Analysis of human cytomegalovirus-encoded SUMO targets and temporal regulation of SUMOylation of the immediate-early proteins IE1 and IE2 during infection. PLoS ONE 2014, 9, e103308. [CrossRef]

40. Liang, C.; Li, M.; Dai, X.; Zhao, S.; Hou, Y.; Zhang, Y.; Lan, D.; Wang, Y.; Chen, X. Autographa californica multiple nucleopolyhedrovirus PK-1 is essential for nucleocapsid assembly. Virology 2013, 443, 349-357. [CrossRef]

41. Mishra, G.; Chadha, P.; Das, R.H. Serine/threonine kinase (pk-1) is a component of Autographa californica multiple nucleopolyhedrovirus (AcMNPV) very late gene transcription complex and it phosphorylates a $102 \mathrm{kDa}$ polypeptide of the complex. Virus Res. 2008, 137, 147-149. [CrossRef] [PubMed]

42. Lu, J.; Wu, T.; Zhang, B.; Liu, S.; Song, W.; Qiao, J.; Ruan, H. Types of nuclear localization signals and mechanisms of protein import into the nucleus. Cell Commun. Signal. 2021, 19, 60. [CrossRef] [PubMed]

43. Han, Q.; Chang, C.; Li, L.; Klenk, C.; Cheng, J.; Chen, Y.; Xia, N.; Shu, Y.; Chen, Z.; Gabriel, G.; et al. Sumoylation of influenza A virus nucleoprotein is essential for intracellular trafficking and virus growth. J. Virol. 2014, 88, 9379-9390. [CrossRef] [PubMed]

44. Way, G.; Xiong, Z.; Wang, G.; Dai, H.; Zheng, S.; Garcia-Sastre, A.; Liao, J. A novel SUMOylation site in the influenza a virus NS1 protein identified with a highly sensitive FRET assay. J. Biotechnol. 2020, 323, 121-127. [CrossRef]

45. Conde, J.N.; Schutt, W.R.; Mladinich, M.; Sohn, S.Y.; Hearing, P.; Mackow, E.R. NS5 Sumoylation Directs Nuclear Responses That Permit Zika Virus To Persistently Infect Human Brain Microvascular Endothelial Cells. J. Virol. 2020, 94, e01086-20. [CrossRef] 